\title{
Set Variables and Local Search
}

\author{
Magnus Ågren \\ Department of Information Technology \\ Uppsala University, Box 337, S - 75105 Uppsala, Sweden \\ agren@it.uu.se
}

\begin{abstract}
Many combinatorial (optimisation) problems have natural models based on, or including, set variables and set constraints. This modelling device has been around for quite some time in the constraint programming area, and proved its usefulness in many applications. This paper introduces set variables and set constraints also in the local search area. It presents a way of representing set variables in the local search context, where we deal with concepts like transition functions, neighbourhoods, and penalty costs. Furthermore, some common set constraints and their penalty costs are defined. These constraints are later used to model three problems and some initial experimental results are reported.
\end{abstract}

\section{Introduction}

For some time now, set variables and set constraints have been around in the Constraint Programming $(C P)$ area. Most of the popular CP systems of today have features for modelling problems using set variables, i.e., variables taking values that are subsets of some universe. Consider the work by Gervet $[6,7]$, Müller and Müller [13], and Puget [16].

Indeed, many problems have natural models using set variables. Classical examples include the set partitioning problem and the set covering problem. However, such problems also appear frequently as sub-problems in many reallife applications. Examples include airline crew rostering, tournament scheduling, time-tabling, and nurse rostering.

To be able to reason about these problems at the higher level that set variables mean may reduce the development time and increase the understanding of an application considerably. Since this is already known from the CP area, we believe that it should be put also in a local search framework.

Local search is becoming more and more popular as an alternative or complement to $\mathrm{CP}$ when it comes to tackling hard combinatorial problems such as the Constraint Satisfaction Problem (CSP). It has proven to be very efficient and often outperforms other techniques $[4,1,15]$.

In recent years, there has been much research on the integration of $\mathrm{CP}$ techniques and local search, investigating such concepts as high declarativeness, incrementality, and global constraints (see [10,14, 5, 3, 11, 17, 12, 2] for instance).

In the following, Section 2 presents the Finite Domain CSP in a local search setting. We define the concepts of configurations, neighbourhoods, and penalty 
costs. Section 3 generalises these concepts for Set CSPs. Then, in Section 4, we introduce some constraints on set variables. We present their semantics and their penalty costs. Section 5 briefly discusses the search component in local search, and some commonly used heuristics. Section 6 presents set-based models for three problems and Section 7 reports on computational results for one of these. Finally, Section 8 contains a discussion about the methods and the results, and Section 9 concludes the paper.

\section{Local Search for Finite Domain CSPs}

A local search algorithm for solving a combinatorial (optimisation) problem $P$ starts from some initial configuration $k$ of $P$ and, using the penalty costs of $P$ with respect to $k$ and the neighbours of $k$, moves to neighbours having less penalty cost. The algorithm stops and returns the current configuration when an optimal penalty cost is obtained, or when some maximum number of iterations is reached. In this section, we define a class of problems, FDCSPs, that local search applies to. We also define the concepts above such as configurations, neighbours, and penalty costs for these problems.

Definition 1. A Finite Domain CSP (FDCSP) is a triple $\left\langle V_{f d}, D_{f d}, C\right\rangle$ where

- $V_{f d}=\left\{v_{1}, \ldots, v_{n}\right\}$ is a finite set of variables.

- $D_{f d}=\left\{D_{1}, \ldots, D_{n}\right\}$ is a finite set of finite domains, each $D_{i}$ containing the set of possible values for the corresponding variable $v_{i}$.

$-C=\left\{c_{1}, \ldots, c_{m}\right\}$ is a finite set of constraints, each $c_{i}$ being defined on a subset of the variables in $V_{f d}$ specifying the valid combinations of values for those.

Definition 2. Let $P=\left\langle V_{f d}, D_{f d}, C\right\rangle$ be an FDCSP. A configuration for $P$ is a function $k: V_{f d} \rightarrow D_{1} \cup \cdots \cup D_{n}$ with the condition that $k\left(v_{i}\right) \in D_{i}$ for all $v_{i} \in V_{f d}$.

Example 1. Let $P=\left\langle\left\{v_{1}, v_{2}, v_{3}\right\},\left\{D_{1}, D_{2}, D_{3}\right\},\left\{c_{1}, c_{2}\right\}\right\rangle$ be an FDCSP where $D_{1}=D_{2}=D_{3}=\{1,2\}$. One possible configuration $k$ for $P$ is defined as $k\left(v_{1}\right)=$ $k\left(v_{2}\right)=1, k\left(v_{3}\right)=2$ or equivalently as the set $k=\left\{v_{1} \mapsto 1, v_{2} \mapsto 1, v_{3} \mapsto 2\right\}$.

Definition 3. Let $K$ denote the set of all possible configurations for an FDCSP $P$. A transition function for $P$ is a function trans $: K \rightarrow 2^{K}$.

Definition 4. Let $P$ be an FDCSP, let $k$ be a configuration for $P$, and let trans be a transition function for $P$. The neighbourhood of $P$ with respect to $k$ and trans is the set of configurations trans $(k)$.

Example 2. Consider $P$ and $k$ from Example 1. A possible neighbourhood $\operatorname{trans}(k)$ of $P$ with respect to some transition function trans is the set of configurations $\left\{\left\{v_{1} \mapsto 2, v_{2} \mapsto 1, v_{3} \mapsto 2\right\},\left\{v_{1} \mapsto 1, v_{2} \mapsto 2, v_{3} \mapsto 2\right\},\left\{v_{1} \mapsto 1, v_{2} \mapsto 1, v_{3} \mapsto\right.\right.$ $1\}\}$. 
Example 3. While the neighbourhood of Example 2 was specified given a particular configuration, we define more general neighbourhoods as shown below. These define a set of configurations given any configuration $k$ of some FDCSP $\left\langle V_{f d}, D_{f d}, C\right\rangle$.

$$
\begin{gathered}
\text { Flip }(k)=\left\{\begin{array}{c}
k^{\prime} \mid \exists v_{i} \in V_{f d}: \exists d \neq d^{\prime} \in D_{i}: k-k^{\prime}=\{v \mapsto d\} \& \\
\left.k^{\prime}-k=\left\{v \mapsto d^{\prime}\right\}\right\}
\end{array}\right. \\
\operatorname{Swap}(k)=\left\{\begin{array}{r}
k^{\prime} \mid \exists v_{i} \neq v_{j} \in V_{f d}: \exists d \neq d^{\prime} \in D_{i}: \exists d \neq d^{\prime} \in D_{j}: \\
\left.k-k^{\prime}=\left\{v_{i} \mapsto d, v_{j} \mapsto d^{\prime}\right\} \& k^{\prime}-k=\left\{v_{i} \mapsto d^{\prime}, v_{j} \mapsto d\right\}\right\}
\end{array}\right.
\end{gathered}
$$

A member of the set Flip $(k)$ differs from $k$ in the value of one variable. A member of the set $\operatorname{Swap}(k)$ differs from $k$ in the values of two variables, where their values have been swapped. The neighbourhood given in Example 2 is an example of a Flip neighbourhood.

Definition 5. Let $P=\left\langle V_{f d}, D_{f d}, C\right\rangle$ be an FDCSP and remember that $K$ denotes the set of all possible configurations for $P$. The penalty cost function of a constraint $c \in C$ is a function $\operatorname{cost}(c): K \rightarrow \mathbb{N}$.

Definition 6. Let $P=\left\langle V_{f d}, D_{f d}, C\right\rangle$ be an FDCSP and let $k$ be a configuration for $P$. The penalty cost of $P$ with respect to $k$ is the sum:

$$
\sum_{c \in C} \operatorname{cost}(c)(k)
$$

Example 4. Consider once again $P$ from Example 1 and let $c_{1}$ and $c_{2}$ be the binary constraints $v_{1}=v_{2}$ and $v_{2} \neq v_{3}$ respectively. Let the penalty cost functions of $c_{1}$ and $c_{2}$ be defined as:

$$
\operatorname{cost}\left(c_{1}\right)=\left\{\begin{array}{l}
0, \text { if } v_{1}=v_{2} \\
1, \text { otherwise }
\end{array} \quad \text { and } \operatorname{cost}\left(c_{2}\right)=\left\{\begin{array}{l}
0, \text { if } v_{2} \neq v_{3} \\
1, \text { otherwise }
\end{array}\right.\right.
$$

Now, the penalty costs of $P$ with respect to the different configurations

$$
\begin{aligned}
& k_{1}=\left\{v_{1} \mapsto 2, v_{2} \mapsto 1, v_{3} \mapsto 2\right\}, k_{2}=\left\{v_{1} \mapsto 1, v_{2} \mapsto 2, v_{3} \mapsto 2\right\}, \text { and } \\
& k_{3}=\left\{v_{1} \mapsto 1, v_{2} \mapsto 1, v_{3} \mapsto 1\right\}
\end{aligned}
$$

are $\operatorname{cost}\left(c_{1}\right)\left(k_{1}\right)+\operatorname{cost}\left(c_{2}\right)\left(k_{1}\right)=1+0=1, \operatorname{cost}\left(c_{1}\right)\left(k_{2}\right)+\operatorname{cost}\left(c_{2}\right)\left(k_{2}\right)=1+1=2$, and $\operatorname{cost}\left(c_{1}\right)\left(k_{3}\right)+\operatorname{cost}\left(c_{2}\right)\left(k_{3}\right)=0+1=1$ respectively.

\section{Local Search for Set CSPs}

Let us now generalise the concepts introduced in Section 2 for another, extended, class of problems.

Definition 7. $A$ Set CSP (SCSP) is a triple $\left\langle\left\langle V_{f d}, V_{\text {set }}\right\rangle,\left\langle D_{f d}, D_{\text {set }}\right\rangle, C\right\rangle$ where 
- $V_{f d}=\left\{v_{1}, \ldots, v_{m}\right\}$ and $V_{\text {set }}=\left\{s_{1}, \ldots, s_{n}\right\}$ are finite sets of variables. $A$ member $v \in V_{f d}$ denotes a finite domain variable, and a member $s \in V_{\text {set }}$ denotes a finite set variable.

- $D_{f d}=\left\{D_{1}, \ldots, D_{m}\right\}$ is a finite set of finite domains, each $D_{i}$ containing the set of possible values for the corresponding variable $v_{i}$.

- $D_{\text {set }}=\left\{U_{1}, \ldots, U_{n}\right\}$ is a finite set of finite sets, each $U_{i}$ being a universe, defined below, for the corresponding variable $s_{i}$.

- $C=\left\{c_{1}, \ldots, c_{m}\right\}$ is a finite set of constraints, each $c_{i} \in C$ being defined on a subset of the variables in $V_{f d} \cup V_{\text {set }}$, specifying the valid combinations of values for those.

Definition 8. Let $s$ be a finite set variable. The universe of $s$ is a finite set $U$ such that $s \subseteq U$.

Definition 9. Let $s$ be a finite set variable and let $U$ be the universe of $s$. The characteristic function of $s$ is the function $\chi_{s}: U \rightarrow\{0,1\}$ defined as:

$$
\chi_{s}(v)= \begin{cases}1, & \text { if } v \in s \\ 0, & \text { otherwise }\end{cases}
$$

Definition 10. Let $P=\left\langle\left\langle V_{f d}, V_{\text {set }}\right\rangle,\left\langle D_{f d}, D_{\text {set }}\right\rangle, C\right\rangle$ be an SCSP. A configuration for $P$ is a pair of functions

$$
\left\langle k_{f d}: V_{f d} \rightarrow D_{1} \cup \cdots \cup D_{m}, k_{\text {set }}: V_{\text {set }} \rightarrow \chi\right\rangle
$$

where $\chi$ is a set of characteristic functions for the variables in $V_{\text {set }}$. A condition for $k_{f d}$ is that $k_{f d}\left(v_{i}\right) \in D_{i}$ for all $v_{i} \in V_{f d}$. Similarly, a condition for $k_{\text {set }}$ is that $\left\{u \mid k_{\text {set }}\left(s_{i}\right)(u)=1\right\} \subseteq U_{i}$ for all $s_{i} \in V_{\text {set }}$.

From the above, we may conclude that the value of a finite set variable $s$ with respect to its universe $U$ and a current configuration $\left\langle k_{f d}, k_{s e t}\right\rangle$ is the set $\left\{u \in U \mid k_{\text {set }}(s)(u)=1\right\}$. Let us now look at an example of a configuration for a specific SCSP.

Example 5. Consider an SCSP $P=\left\langle\left\langle\left\{v_{1}, v_{2}\right\},\left\{s_{1}, s_{2}\right\}\right\rangle,\left\langle\left\{D_{1}, D_{2}\right\},\left\{U_{1}, U_{2}\right\}\right\rangle\right.$, $C\rangle$ where $D_{1}=D_{2}=\{1,2\}$ and $U_{1}=U_{2}=\{a, b, c\}$. Let $\chi_{1}$ and $\chi_{2}$ be the sets of mappings $\{a \mapsto 0, b \mapsto 0, c \mapsto 1\}$ and $\{a \mapsto 1, b \mapsto 1, c \mapsto 0\}$ respectively.

One possible configuration $\left\langle k_{f d}, k_{\text {set }}\right\rangle$ for $P$ is defined as $k=k_{f d}\left(v_{1}\right)=$ $1, k_{f d}\left(v_{2}\right)=1, k_{s e t}\left(s_{1}\right)=\chi_{1}, k_{s e t}\left(s_{2}\right)=\chi_{2}$ or equivalently as the pair of sets $\left\langle\left\{v_{1} \mapsto 1, v_{2} \mapsto 1\right\},\left\{s_{1} \mapsto \chi_{1}, s_{2} \mapsto \chi_{2}\right\}\right\rangle$. Under this configuration, the values of the variables in $P$ are $v_{1}=1, v_{2}=1, s_{1}=\{c\}$, and $s_{2}=\{a, b\}$.

Example 6. Consider $P$ and $k$ from Example 5 and let trans be a transition function for $P$. A possible neighbourhood for $P$ with respect to $k$ and trans is the set of configurations trans $(k)=\left\{\left\langle\left\{v_{1} \mapsto 1, v_{2} \mapsto 1\right\},\left\{s_{1} \mapsto \chi_{1}, s_{2} \mapsto\right.\right.\right.$ $\left.\left.\left.\chi_{2}\right\}\right\rangle,\left\langle\left\{v_{1} \mapsto 1, v_{2} \mapsto 1\right\},\left\{s_{1} \mapsto \chi_{3}, s_{2} \mapsto \chi_{4}\right\}\right\rangle\right\}$ where

$$
\begin{aligned}
& \chi_{1}=\{a \mapsto 1, b \mapsto 0, c \mapsto 0\}, \chi_{2}=\{a \mapsto 0, b \mapsto 1, c \mapsto 1\}, \\
& \chi_{3}=\{a \mapsto 0, b \mapsto 1, c \mapsto 0\}, \text { and } \chi_{4}=\{a \mapsto 1, b \mapsto 0, c \mapsto 1\} .
\end{aligned}
$$


This neighbourhood does not affect the finite domain variables of the problem but flips any two values in the finite set variables $s_{1}$ and $s_{2}$.

Example 7. As we did for FDCSPs, we will define general neighbourhoods also for SCSPs. Let us do this for an SCSP $\left\langle\left\langle V_{f d}, V_{\text {set }}\right\rangle,\left\langle D_{f d}, D_{\text {set }}\right\rangle, C\right\rangle$ and a configuration $\left\langle k_{f d}, k_{s e t}\right\rangle$.

$$
\begin{aligned}
\operatorname{trans}\left(\left\langle k_{f d}, k_{\text {set }}\right\rangle\right)=\left\{\left\langle k_{f d}, k\right\rangle \mid\right. & k_{\text {set }}-k=\{s \\
k_{\text {set }}(s)-k(s) & =\left\{u \mapsto \chi_{s}\right\} \& \\
k(s)-k_{\text {set }}(s) & \left.\left.=\left\{u \mapsto 0, u^{\prime} \mapsto 0\right\} \& 1\right\}\right\}
\end{aligned}
$$

The above neighbourhood leaves $k_{f d}$ untouched. $k_{\text {set }}$ and $k$ differ in exactly one mapping $s \mapsto \chi_{s}$. For this variable, the characteristic function $k_{\text {set }}(s)$ differs from $k(s)$ in exactly two values, say $u$ and $u^{\prime}$. For $k_{\text {set }}, u \in s$ and $u^{\prime} \notin s$, while the opposite is true for $k$. We have thus defined a neighbourhood that flips one value in one finite set variable.

Let us generalise the neighbourhood presented in the above example and also present additional useful neighbourhoods for SCSPs. We focus here on neighbourhoods that change the finite set variables of the problem and do nothing to the finite domain variables. One could of course also consider neighbourhoods that change both sets of variables.

Example 8. Roughly, there are five different things one can do with a finite set variable: 1 . Add $p$ values to it taken from its complement, increasing its cardinality. 2. Remove $p$ values from it, decreasing its cardinality. 3. Flip $p$ values in it with values from its complement. 4 . Swap $p$ values in it to values in another finite set variable. 5. Any combination of the previous. The first four of these, with respect to the SCSP and configuration of Example 7, are shown below.

$$
\begin{aligned}
& A d d P\left(\left\langle k_{f d}, k_{s e t}\right\rangle\right)=\left\{\left\langle k_{f d}, k\right\rangle \mid k_{s e t}-k=\left\{s \mapsto \chi_{s}\right\} \&\right. \\
& k_{\text {set }}(s)-k(s)=\left\{v_{1} \mapsto 0, \ldots, v_{p} \mapsto 0\right\} \quad \& \\
& \left.k(s)-k_{\text {set }}(s)=\left\{v_{1} \mapsto 1, \ldots, v_{p} \mapsto 1\right\}\right\} \\
& \operatorname{Remove} P\left(\left\langle k_{f d}, k_{\text {set }}\right\rangle\right)=\left\{\left\langle k_{f d}, k\right\rangle \mid k_{\text {set }}-k=\left\{s \mapsto \chi_{s}\right\} \&\right. \\
& k_{\text {set }}(s)-k(s)=\left\{v_{1} \mapsto 1, \ldots, v_{p} \mapsto 1\right\} \quad \& \\
& \left.k(s)-k_{\text {set }}(s)=\left\{v_{1} \mapsto 0, \ldots, v_{p} \mapsto 0\right\}\right\} \\
& \operatorname{FlipP}\left(\left\langle k_{f d}, k_{\text {set }}\right\rangle\right)=\left\{\left\langle k_{f d}, k\right\rangle \mid k_{\text {set }}-k=\left\{s \mapsto \chi_{s}\right\} \&\right. \\
& k_{\text {set }}(s)-k(s)=\left\{v_{1} \mapsto 1, \ldots, v_{p} \mapsto 1, v_{1}^{\prime} \mapsto 0, \ldots, v_{p}^{\prime} \mapsto 0\right\} \quad \& \\
& \left.k(s)-k_{\text {set }}(s)=\left\{v_{1} \mapsto 0, \ldots, v_{p} \mapsto 0, v_{1}^{\prime} \mapsto 1, \ldots, v_{p}^{\prime} \mapsto 1\right\}\right\} \\
& \operatorname{Swap} P\left(\left\langle k_{f d}, k_{\text {set }}\right\rangle\right)=\left\{\left\langle k_{f d}, k\right\rangle \mid k_{\text {set }}-k=\left\{s \mapsto \chi_{s}, s^{\prime} \mapsto \chi_{s^{\prime}}\right\} \&\right. \\
& k_{\text {set }}(s)-k(s)=\left\{v_{1} \mapsto 1, \ldots, v_{p} \mapsto 1, v_{1}^{\prime} \mapsto 0, \ldots, v_{p}^{\prime} \mapsto 0\right\} \quad \& \\
& k_{\text {set }}\left(s^{\prime}\right)-k\left(s^{\prime}\right)=\left\{v_{1} \mapsto 0, \ldots, v_{p} \mapsto 0, v_{1}^{\prime} \mapsto 1, \ldots, v_{p}^{\prime} \mapsto 1\right\} \\
& k(s)-k_{\text {set }}(s)=\left\{v_{1} \mapsto 0, \ldots, v_{p} \mapsto 0, v_{1}^{\prime} \mapsto 1, \ldots, v_{p}^{\prime} \mapsto 1\right\} \\
& \left.k\left(s^{\prime}\right)-k_{\text {set }}\left(s^{\prime}\right)=\left\{v_{1} \mapsto 1, \ldots, v_{p} \mapsto 1, v_{1}^{\prime} \mapsto 0, \ldots, v_{p}^{\prime} \mapsto 0\right\}\right\}
\end{aligned}
$$

Note that the neighbourhood FlipP is a generalisation of the one presented in Example 7. Also, while these are generic neighbourhoods, applicable to any model of an SCSP, one could also think of more problem specific neighbourhoods that depend on the actual model. This is discussed further in Section 6.4. 


\section{Constraints and Penalty Costs}

In this section, we present five constraints on finite set variables: the AllDisjoint constraint, the Cardinality constraint, the Intersection constraint, the MaxSum constraint, and the Partition constraint. We discuss their semantics and penalty costs at a high and abstract level.

Concerning penalty costs, we have followed the approach taken for instance in [5]; the penalty cost for a constraint $c$ is, whenever possible, the least number of values that must change in order to satisfy $c$. This means that, when there is a choice between adding values to, removing values from, or swapping values between the variables in $c$, we will assume the operation resulting in the minimal cost.

\subsection{The AllDisjoint Constraint.}

The constraint allDisjoint $\left(\left\{s_{1}, \ldots, s_{n}\right\}\right)$, where $\left\{s_{1}, \ldots, s_{n}\right\}$ is a set of finite set variables, expresses that all these variables are disjoint, i.e., $\forall i \neq j \in 1 \ldots n$ : $s_{i} \cap s_{j}=\emptyset$.

The penalty cost for an allDisjoint $\left(\left\{s_{1}, \ldots, s_{n}\right\}\right)$ constraint is equal to the number of values that must be removed from any of the variables in $\left\{s_{1}, \ldots, s_{n}\right\}$ in order to make them all disjoint. This is equal to the sum

$$
\sum_{v \in s_{1} \cup \cdots \cup s_{n}}\left(v_{o c c}-1\right)
$$

where $v_{\text {occ }}$ is the number of variables in $\left\{s_{1}, \ldots, s_{n}\right\}$ that contain $v$. Clearly, we need to remove a value from $n-1$ finite set variables if it is contained in $n$ finite set variables in order to satisfy the constraint.

Next, let us show that a satisfied allDisjoint constraint has zero penalty cost. A satisfied instance allDisjoint $\left(\left\{s_{1}, \ldots, s_{n}\right\}\right)$ of this constraint has the property that $\forall i \neq j \in 1 \ldots n: s_{i} \cap s_{j}=\emptyset$. This implies that for each $v \in s_{1} \cup \cdots \cup s_{n}$ we have that $v_{o c c}=1$. Hence, the equation (1) above must be equal to 0 .

The following example illustrates the above. Assume that $s_{1}=\{1,2,3\}, s_{2}=$ $\{1,2,4\}$, and $s_{3}=\{1,4\}$. The values $1,2,3$, and 4 , that comprise the union of $s_{1}$, $s_{2}$, and $s_{3}$, appear in $3,2,1$, and 2 variables respectively. Thus, the above sum and the penalty cost for allDisjoint $\left(\left\{s_{1}, s_{2}, s_{3}\right\}\right)$ is $(3-1)+(2-1)+(1-1)+(2-1)=4$.

\subsection{The Cardinality Constraint.}

The constraint $\operatorname{card}\left(s, v_{\min }, v_{\max }\right)$, where $s$ is a finite set variable and $v_{\min }$ and $v_{\max }$ are finite integer domain variables, expresses that the cardinality of $s$ is in the range $v_{\min } \ldots v_{\max }$.

The penalty cost for a $\operatorname{card}\left(s, v_{\min }, v_{\max }\right)$ constraint is equal to the number of values that must be added to or removed from $s$ so that its cardinality is in the range $v_{\min } \ldots v_{\max }$. This is calculated as follows. If $|s|$ is less than $v_{\min }$, the penalty cost of the constraint is $v_{\text {min }}-|s|$. If $|s|$ is larger than $v_{\max }$, the penalty 
cost of the constraint is $|s|-v_{\max }$. Otherwise, the penalty cost of the constraint is 0 . Hence

$$
\text { CardCost }=\left\{\begin{array}{l}
v_{\min }-|s|, \text { if }|s|<v_{\text {min }} \\
|s|-v_{\max }, \text { if }|s|>v_{\text {max }} \\
0, \text { otherwise }
\end{array}\right.
$$

denotes the penalty cost of this constraint. Now, to see that a satisfied instance of this constraint has zero penalty cost is trivial.

Now, assume that the current value of $s$ is $\{1,2,3,4,5\}$, that $v_{\min }=1$, and that $v_{\max }=3$. Since the cardinality of $s$ is 5 and $v_{\max }=3$, we need to remove at least $5-3=2$ values from $s$ in order to make the constraint satisfied.

\subsection{The Intersection Constraint.}

The constraint intersect $\left(s_{1}, s_{2}, s\right)$, where $s_{1}, s_{2}$, and $s$ are finite set variables, expresses that the intersection of $s_{1}$ and $s_{2}$ is equal to $s$, i.e., that $s_{1} \cap s_{2}=s$.

The penalty cost for an intersect $\left(s_{1}, s_{2}, s\right)$ constraint is equal to the least number of values that must be removed from, added to, or swapped between any of the variables $s_{1}, s_{2}$, and $s$ so that $s_{1} \cap s_{2}=s$. This is calculated in four steps as follows. First, the set of values that are in $s_{1}$ and $s_{2}$ but not in $s$ is identified. This set should be removed from both $s_{1}$ and $s_{2}$, or added to $s$. Second, the set of values that are in $s$ but not in any of $s_{1}$ and $s_{2}$ is identified. This set should be removed from $s$, or added to both $s_{1}$ and $s_{2}$. Third, the set of values that are in $s$ and $s_{1}$ but not in $s_{2}$ is identified. This set should be added to $s_{2}$, or removed from $s$. Fourth, the set of values that are in $s$ and $s_{2}$ but not in $s_{1}$ is identified. This set should be added to $s_{1}$, or removed from $s$. From this, we obtain the following sum expressing the penalty cost for the constraint:

$$
\left|\left(s_{1} \cap s_{2}\right)-s\right|+\left|s-\left(s_{1} \cup s_{2}\right)\right|+\left|\left(s \cap s_{1}\right)-s_{2}\right|+\left|\left(s \cap s_{2}\right)-s_{1}\right|
$$

In fact, we can improve on the above by observing the following. For the identified set of values, say $s_{a}$ and $s_{b}$, in the first and second step, we have decided to respectively add these to and remove these from $s$ when calculating the penalty cost. Since $s_{b}$ is to be removed from $s$ and $s_{a}$ is to be added to $s$, we may instead swap values from $s_{b}$ to values in $s_{a}$. This is true for the first and third step and the first and fourth step as well. By doing this whenever possible, i.e., for a maximum of $\left|s_{a}\right|=\left|\left(s_{1} \cap s_{2}\right)-s\right|$ values, we obtain the following new expression for the penalty cost:

$$
\begin{gathered}
\left|\left(s_{1} \cap s_{2}\right)-s\right|+\left|s-\left(s_{1} \cup s_{2}\right)\right|+\left|\left(s \cap s_{1}\right)-s_{2}\right|+\left|\left(s \cap s_{2}\right)-s_{1}\right|- \\
\min \left(\left|\left(s_{1} \cap s_{2}\right)-s\right|,\left|\left(s-\left(s_{1} \cup s_{2}\right)\right) \cup\left(\left(s \cap s_{1}\right)-s_{2}\right) \cup\left(\left(s \cap s_{2}\right)-s_{1}\right)\right|\right)
\end{gathered}
$$

Now, let us show that a satisfied instance intersect $\left(s_{1}, s_{2}, s\right)$ of this constraint has zero penalty cost. For such an instance, we have that $s_{1} \cap s_{2}=s$. In the expression (4) above, the first term is 0 since all elements in $s$ appear also in the intersection of $s_{1}$ and $s_{2}$. The second term is 0 since all elements in $s$ appear also 
in $s_{1}$ or $s_{2}$. The third term is 0 since the intersection of $s$ and $s_{1}$ cannot contain an element that is not in $s_{2}$. A similar reasoning holds for the fourth term. The fifth term is also 0 by the above and hence we obtain a total penalty cost of 0 .

As an example, assume that $s_{1}=\{1,2,3,4,5\}, s_{2}=\{4,5,6,7,8\}$, and $s=$ $\{1,2,3,6,7\}$. The values 4 and 5 in the intersection of $s_{1}$ and $s_{2}$ are not in $s$. Also, the values 1,2 , and 3 are in the intersection of $s$ and $s_{1}$ but are not in $s_{2}$. Similarly, the values 6 and 7 are in the intersection of $s$ and $s_{2}$ but are not in $s_{1}$. Finally, the fifth term in expression (4) evaluates to 2 . This results in a penalty cost of $2+0+3+2-2=5$.

\subsection{The MaxSum Constraint.}

Let $s$ be a finite set variable, weight $: U \rightarrow \mathbb{N}$ a weight function from the universe $U$ of $s$ to the integers, and $m$ a finite integer domain variable. The constraint $\operatorname{maxSum}(s$, weight, $m)$ expresses that the sum $\sum_{u \in s}$ weight $(u)$ is less than or equal to $m$.

The penalty cost for a $\operatorname{maxSum}(s$, weight, $m)$ constraint, where we let Sum denote the sum $\sum_{u \in s}$ weight $(u)$, is equal to the least number of values that must be removed from $s$ in order for Sum to be less than or equal to $m$. This penalty cost is calculated by finding the minimal subset $s^{\prime}$ of values of $s$ such that the sum $\sum_{u^{\prime} \in s^{\prime}}$ weight $\left(u^{\prime}\right)$ is larger than or equal to the difference between Sum and $m$. By removing the set $s^{\prime}$ from $s$ we will obtain a new sum $S_{u m}^{\prime}$ that is less than or equal to $m$. Hence, the expression:

$$
\left|\min \left(\left\{s^{\prime} \subseteq s \mid \sum_{u^{\prime} \in s^{\prime}} \operatorname{weight}\left(u^{\prime}\right) \geq\left(\sum_{u \in s} \operatorname{weight}(u)\right)-m\right\}\right)\right|
$$

denotes the penalty cost for this constraint, where $\min \left(\left\{s_{1}, \ldots, s_{n}\right\}\right)$ denotes a set $s_{i}$ with minimal cardinality among $s_{1}, \ldots, s_{n}$.

Now, let us show that a satisfied instance $\max \operatorname{Sum}(s$, weight, $m)$ of this constraint has zero penalty cost. For such an instance, we have that the sum $\sum_{u \in s}$ weight $(u)$ is less than or equal to $m$. This means that the difference $d$ between these in expression (5) is less than or equal to 0 . Then we need to find the minimal subset $s^{\prime}$ of $s$ such that $\sum_{u^{\prime} \in s^{\prime}}$ weight $\left(u^{\prime}\right) \geq d$. Clearly, the least such subset of $s$ is $\emptyset$ and $|\emptyset|=0$.

As an example, let $s=\{1,2,3\}$, weight $(1)=2$, weight $(2)=\operatorname{weight}(3)=1$, and $m=2$. Then the sum $\sum_{u \in s}$ weight $(u)=4$. In order to satisfy this constraint, the least subset of values to remove from $s$ is $\{1\}$ since weight(1) $=2 \geq 4-2$ and there is no smaller subset $s^{\prime} \subseteq s$ such that $\sum_{u \in s^{\prime}}$ weight $(u) \geq 2$. Hence the penalty cost of this constraint is 1 .

\subsection{The Partition Constraint.}

The constraint partition $\left(\left\{s_{1}, \ldots, s_{n}\right\}, s\right)$, where $\left\{s_{1}, \ldots, s_{n}\right\}$ is a set of finite set variables and $s$ is a finite set variable, expresses that the variables in $\left\{s_{1}, \ldots, s_{n}\right\}$ 
are all disjoint, i.e., that $\forall i \neq j \in 1 \ldots n: s_{i} \cap s_{j}=\emptyset$, and that their union is equal to $s$, i.e., that $s_{1} \cup \cdots \cup s_{n}=s$. Note that this definition of a partition allows one or more variables in $\left\{s_{1}, \ldots, s_{n}\right\}$ to be empty.

The penalty cost for a partition $\left(\left\{s_{1}, \ldots, s_{n}\right\}, s\right)$ constraint is equal to the number of values that must be removed from any variable in $\left\{s_{1}, \ldots, s_{n}\right\}$ in order to make them all disjoint plus the number of values that must be removed from, added to, or swapped between any of the variables in $\left\{s, s_{1}, \ldots, s_{n}\right\}$ so that the union $s_{1} \cup \cdots \cup s_{n}$ is equal to $s$.

We start by calculating the penalty cost for the overlapping values among the variables $s_{1}, \ldots, s_{n}$. This is done in the same way as for the allDisjoint constraint, i.e., the expression (1) denotes this penalty cost. Next, we calculate the penalty cost for the values in $s_{1} \cup \cdots \cup s_{n}$ that are not in $s$. These values must be removed from $s_{1} \cup \cdots \cup s_{n}$, or added to $s$. Finally, we calculate the penalty cost for the values in $s$ that are not in $s_{1} \cup \cdots \cup s_{n}$. These values must be removed from $s$, or added to $s_{1} \cup \cdots \cup s_{n}$. Now, by following the same reasoning as for the intersect constraint, we see that a number of values in $s$ and $s_{1} \cup \cdots \cup s_{n}$ can be swapped directly, giving us a subtrahend in our penalty cost expression. Hence, the expression:

$$
\begin{gathered}
\left(\sum_{u \in s_{1} \cup \cdots \cup s_{n}}\left(v_{o c c}-1\right)\right)+\left|\left(s_{1} \cup \cdots \cup s_{n}\right)-s\right|+\left|s-\left(s_{1} \cup \cdots \cup s_{n}\right)\right|- \\
\min \left(\left|\left(s_{1} \cup \cdots \cup s_{n}\right)-s\right|,\left|s-\left(s_{1} \cup \cdots \cup s_{n}\right)\right|\right)
\end{gathered}
$$

denotes the penalty cost for the constraint.

Now we show that a satisfied instance partition $\left(\left\{s_{1}, \ldots, s_{n}\right\}, s\right)$ of this constraint has zero penalty cost. We know from the allDisjoint constraint that, for a satisfied instance, the first term in expression (6) above is 0. For the second and third term, note that for a satisfied instance of this constraint we have that $s_{1} \cup \cdots \cup s_{n}=s$. The difference between two equal sets is the empty set $\emptyset$. Hence, the last two terms are 0 , giving us a total penalty cost of 0 .

As an example, assume that $s_{1}=\{1,2,3\}, s_{2}=\{2,3,4\}, s_{3}=\{2,5\}$, and that $s=\{4,5,6,7\}$. First, in order for $s_{1}, s_{2}$, and $s_{3}$ to be disjoint, we need to remove the value 2 from two of the variables in $\left\{s_{1}, s_{2}, s_{3}\right\}$ and the value 3 from one of the variables in $\left\{s_{1}, s_{2}\right\}$. Second, in order for $s_{1} \cup s_{2} \cup s_{3}$ to be a subset of $s$, we need to remove 1,2 , and 3 from that union, or add these values to $s$. Third, in order for $s$ to be a subset of $s_{1} \cup s_{2} \cup s_{3}$, we need to remove 6 and 7 from $s$, or add these values to the union. Since two of the values in the second and third steps may be swapped directly, we obtain a subtrahend of 2 . This gives us a total penalty cost of $3+3+2-2=6$.

\section{$5 \quad$ Search and Heuristics}

One crucial point when it comes to local search is the ability to avoid getting stuck in local optima. If nothing is done to avoid this, the algorithm will probably perform very badly. Much research has gone into this area and some approaches 
are different randomisation algorithms, simulated annealing, and tabu search. Randomisation algorithms have, in addition to the penalty cost of the current configuration and its neighbours, some random-based way of choosing the next configuration. This means that the algorithm does not always have to move to a configuration having less penalty cost compared to the current one. Simulated annealing [9] is actually a variant of such a randomisation algorithm, in which the probability of choosing a degrading configuration changes over time. This method was derived from the process of growing a crystal in thermodynamics and has proven to be very efficient.

In this paper we have used tabu search [8] in our experiments. Such an algorithm may also move to worse configurations with respect to their penalty costs. When the next move is considered, a configuration with minimal penalty cost among the neighbours is chosen, even though this penalty cost may be larger than the penalty cost of the current configuration. In order to avoid a cyclic behaviour the method uses a memory called tabu list. This list stores configurations from a number, the tabu tenure, of previous iterations and any configuration contained in it is not allowed.

There are many ways to represent the tabu list. We have chosen the following simple approach for the finite set variables. For each value $u \in U$, where $U$ is the universe of a finite set variable $s$, we keep a counter that denotes the number of future iterations where $u$ is tabu. When $s$ is instantiated to a particular value, say given by the characteristic function $\chi_{s}$, the counters for all elements in the set $\left\{u \in U \mid \chi_{S}(u)=1\right\}$ are set to the tabu tenure value. At each iteration, the counters for all values in $U$ greater than 0 are decreased by 1 .

\section{Example Problems}

This section presents possible models using finite set variables and the constraints presented in Section 4 for three different problems: the progressive party problem, the social golfer problem, and the minimal intersection subset problem. It demonstrates the usefulness of having finite set variables as a modelling device.

\subsection{The Progressive Party Problem}

The problem is to timetable a party at a yacht club. Certain boats are designated as hosts, while the crews of the remaining boats are designated as guests. The crew of a host boat remains on board throughout the party to act as hosts, while the crew of a guest boat together visits host boats over a number of periods. The crew of a guest boat must party at some host boat each period $\left(c_{1}\right)$. The spare capacity of any host boat is not to be exceeded at any period by the sum of the crew sizes of all the guest boats that are scheduled to visit it then $\left(c_{2}\right)$. Any guest crew can visit any host boat in at most one period $\left(c_{3}\right)$. Any two distinct guest crews can visit the same host boat in at most one period $\left(c_{4}\right)$. 
Model. Let $H=\left\{h_{1}, \ldots, h_{m}\right\}$ be the set of host boats and let $G=\left\{g_{1}, \ldots, g_{n}\right\}$ be the set of guest boats. Furthermore, let capacity $(h)$ and size $(g)$ denote the spare capacity of host boat $h$ and the crew size of guest boat $g$ respectively. Let periods be the number of periods we want to find a schedule for, and let $P=1 \ldots$ periods be the range of periods. Now, let $p_{(h, j)}$ be a finite set variable containing the set of guest boats whose crews boat $h$ hosts at time period $j$. Then the following constraints model the problem:

$$
\begin{aligned}
& \left(c_{1}\right): \forall j \in P: \operatorname{partition}\left(\left\{p_{\left(h_{1}, j\right)}, \ldots, p_{\left(h_{m}, j\right)}\right\}, G\right) \\
& \left(c_{2}\right): \forall h \in H: \forall j \in P: \operatorname{maxSum}\left(p_{(h, j)}, \operatorname{size}, \operatorname{capacity}(h)\right) \\
& \left(c_{3}\right): \forall h \in H: \forall j \neq j^{\prime} \in P: \operatorname{intersect}\left(p_{(h, j)}, p_{\left(h, j^{\prime}\right)}, \emptyset\right) \\
& \left(c_{4}\right): \forall h \neq h^{\prime} \in H: \forall j \neq j^{\prime} \in P: \operatorname{intersect}\left(p_{(h, j)}, p_{\left(h^{\prime}, j^{\prime}\right)}, s_{\left(h, j, h^{\prime}, j^{\prime}\right)}\right) \quad \& \\
& \quad \operatorname{card}\left(s_{\left(h, j, h^{\prime}, j^{\prime}\right)}, 0,1\right)
\end{aligned}
$$

where the introduced finite set variables $s_{\left(h, j, h^{\prime}, j^{\prime}\right)}$ in $\left(c_{4}\right)$ have $G$ as universe.

\subsection{The Social Golfer Problem}

In a golf club, there are $n$ players, each of whom play golf once a week $\left(c_{1}\right)$ and always in $g$ groups of size $s\left(c_{2}\right)$, hence $n=g s$. The objective is to determine whether there is a schedule of $w$ weeks of play for these golfers, such that there is at most one week where any two distinct players are scheduled to play in the same group $\left(c_{3}\right)$.

Model. Let $P=\left\{p_{1}, \ldots, p_{n}\right\}$ be the set of golfers. Let $g_{(i, j)}$ be a finite set variable containing the players playing in group $i$ in week $j$. Then the following constraints model the problem:

$$
\begin{aligned}
& \left(c_{1}\right): \forall j \in 1 \ldots w: \operatorname{partition}\left(\left\{g_{(1, j)}, \ldots, g_{(g, j)}\right\}, P\right) \\
& \left(c_{2}\right): \forall i \in 1 \ldots g: \forall j \in 1 \ldots w: \operatorname{card}\left(g_{(i, j)}, s, s\right) \\
& \left(c_{3}\right): \forall i, i^{\prime} \in 1 \ldots g: \forall j \neq j^{\prime} \in 1 \ldots w: \operatorname{intersect}\left(g_{(i, j)}, g_{\left(i^{\prime}, j^{\prime}\right)}, s_{\left(i, i^{\prime}, j, j^{\prime}\right)}\right) \quad \& \\
& \quad \operatorname{card}\left(s_{\left(i, i^{\prime}, j, j^{\prime}\right)}, 0,1\right)
\end{aligned}
$$

where the introduced finite set variables $s_{\left(i, i^{\prime}, j, j^{\prime}\right)}$ in $\left(c_{3}\right)$ have $P$ as universe.

\subsection{The Minimal Intersection Subset Problem}

Assume a set $R$ of $r$ elements. The objective is to find $k \geq 2$ subsets of $R$ of size $p\left(c_{1}\right)$ such that the size of the largest intersection between any two different subsets is minimised $\left(c_{2}, c_{3}\right)$.

Model. Let $R=\{1, \ldots, r\}$ and let $r_{i}$ be a finite set variable denoting the $i$ th subset of $R$. Then the following constraints model the problem and the objective is to minimise $\delta$ :

$$
\left(c_{1}\right): \forall i \in 1 \ldots k: \operatorname{card}\left(r_{i}, p, p\right)
$$




$$
\begin{aligned}
& \left(c_{2}\right): \forall i \neq j \in 1 \ldots k: \operatorname{intersect}\left(r_{i}, r_{j}, s_{(i, j)}\right) \& \operatorname{card}\left(s_{(i, j)}, \delta_{(i, j)}, \delta_{(i, j)}\right) \\
& \left(c_{3}\right): \max \left(\left\{\delta_{(i, j)} \mid i \neq j \in 1 \ldots k\right\}, \delta\right)
\end{aligned}
$$

In the above, the introduced finite set variables $S_{(i, j)}$ have $R$ as their universe, the introduced finite integer domain variables in $\{\delta\} \cup\left\{\delta_{i, j} \mid i \neq j \in 1 \ldots k\right\}$ can take values in the domain $0 \ldots r$, and $\max (\operatorname{Set}, m)$ is satisfied iff $m$ takes the same value as the maximum element in Set.

\subsection{Problem-Specific Neighbourhoods}

The models presented above respect any choice of neighbourhood for the finite set variables of the problems. By this, we mean that one may add, remove, flip, or swap values in the variables when defining a neighbourhood and still be sure that a solution to the model is a solution to the modelled problem.

One could also think of more neighbourhood-specific models, i.e., models that do not allow all possible neighbourhoods. This means that the problems can be modelled using fewer constraints, allowing for more efficient solving.

For example, the model for the social golfer problem, presented in Section 6.2, could also be modelled by dropping the constraints in $\left(c_{2}\right)$. These constraints force each finite set variable $g_{(i, j)}$ to have cardinality $s$, i.e., they force the group size constraint. If these constraints are not added to the model, care must be taken when defining neighbourhoods. In this case any neighbourhood that does not change the size of the finite set variables, and an initial assignment respecting $\left(c_{2}\right)$, would be valid. In addition to this, one could also replace the partition constraint in $\left(c_{1}\right)$ by an allDisjoint constraint. If this is done, we need to make sure that any chosen neighbourhood keeps the property that the union of all the finite set variables in each week is equal to the total set of golfers. Of course, the initial assignment would also have to respect this property. Actually, we could even drop the partition constraint completely if we use a neighbourhood that respects it. For instance, a $S w a p P$ neighbourhood that only swaps values between variables in the same week would be a valid one.

\section{$7 \quad$ Results}

Due to lack of space we only present experimental results for the social golfer problem. We compared our set based model for this problem with a model based on finite domain variables. Both models were implemented in Objective Caml (http://www.ocaml.org) and the results are displayed in Table 1.

The finite domain model is based on each group in each week being modelled as a list of size $s$ of finite domain variables. Each such variable has the domain $1 \ldots n$, where $n$ is the total number of golfers, and relevant finite domain constraints for modelling the problem are stated. For this model, we used the neighbourhood Flip as was given in Example 3 of Section 2, i.e., the neighbourhood where the value of any variable is flipped to another value in its domain.

For the finite set variables in the set based model we used the neighbourhood SwapP as was given in Example 8 of Section 3, i.e., the neighbourhood where 
Table 1. Experimental results on the social golfer problem. Numbers displayed in bold correspond to the set based approach.

\begin{tabular}{|c|c|c|c|c|c|c|c|}
\hline $3-3-27$ & 110 & 10 & 7.0 & |5-3-5 98 & 1010 & 10 & 9.8 \\
\hline $3-3-3 \quad 12$ & 210 & 10 & 6.0 & 5-3-6 216 & 681 & 4 & 3.2 \\
\hline $3-3-4 \quad 32$ & 410 & 10 & 8.0 & $5-3-7 ?$ & 1360 & 4 & ? \\
\hline $4-3-2 \quad 9$ & 110 & 10 & 9.0 & $5-4-2 \quad 16$ & 310 & 10 & 5.3 \\
\hline $4-3-3 \quad 18$ & 310 & 10 & 6.0 & $5-4-3 \quad 41$ & 610 & 10 & 6.8 \\
\hline $4-3-4 \quad 91$ & 610 & 10 & 15.2 & $5-4-4271$ & 1910 & 10 & 14.3 \\
\hline $4-4-2 \quad 12$ & 210 & 10 & 6.0 & $5-4-5 \quad 476$ & 911 & 4 & 5.2 \\
\hline $4-4-3 \quad 36$ & 610 & 10 & 6.0 & $8-4-2 \quad 23$ & 210 & 10 & 11.5 \\
\hline $4-4-4226$ & 1610 & 10 & 14.1 & $8-4-3 \quad 37$ & 510 & 10 & 7.4 \\
\hline $4-4-5 \quad 255$ & 257 & 10 & 10.2 & $8-4-458$ & 1010 & 10 & 5.8 \\
\hline $5-3-211$ & 110 & 10 & 11.0 & $8-4-597$ & 1510 & 10 & 6.5 \\
\hline $5-3-3 \quad 18$ & 310 & 10 & 6.0 & $8-4-6208$ & 3310 & 10 & 6.3 \\
\hline $5-3-4 \quad 32$ & 410 & 10 & 8.0 & 8-4-7 698 & 1119 & 10 & 6.3 \\
\hline
\end{tabular}

any two values for any two variables are swapped. This neighbourhood needs an initial assignment respecting the constraints $\left(c_{1}\right)$ and $\left(c_{2}\right)$.

In Table 1, bold numbers correspond to the set variable approach. For both approaches, each instance was run 10 times. For the finite domain based approach, a maximum of 1000 iterations was allowed in each run. When 1000 iterations were reached the instance was considered not solved. For the set based approach, this number was set to 200. The first column displays the instance solved, where $g$ is the number of groups, $s$ is the size of each group, and $w$ is the number of weeks to find a schedule for. The second column displays the average number of iterations needed to find a solution among the runs where a solution was actually found. A non-solved instance was thus not contributing to this value. The third column displays the number of runs, out of 10 , where a solution was found. Finally, the fourth column displays the improvement ratio, i.e., the average number of iterations in the finite domain based model divided by the average number of iterations in the set based model.

\section{Discussion}

As can be seen in Table 1, the number of iterations needed in the model based on set variables is less than the number of iterations needed in the model based on finite domain variables in all instances tried. The set based model also solves instances where the finite domain based model fails in all runs.

One reason for this is probably the different choices of neighbourhoods. The SwapP neighbourhood used in the set based model is more powerful than the simple Flip neighbourhood used in the finite domain based model. However, the neighbourhoods picked are natural for their respective models. Defining a 
neighbourhood similar to the $S w a p P$ one for the finite domain model would be tedious. Having finite set variables at ones disposal simplifies this process considerably.

Another measurement that could be used is processing time. However, the current implementation of the set based constraints uses naïve algorithms for updating the penalty costs when exploring neighbourhoods. No incrementality whatsoever is used which, of course, implies poor performance. This is also seen by looking at the solved instances of the social golfer problem; none of them are particularly hard. Now, the aim of this paper was not (yet) to break any new records, but to present a new way of solving combinatorial (optimisation) problems using local search. Efficiency will come later and there are many options available to achieve this. One could for instance use the approach taken in Comet [11] by introducing invariants in the system, or the approach taken in [2] by reasoning about the constraints from a graph-based point of view.

\section{Conclusion}

We introduced finite set variables into local search. We defined the concepts of configurations, transition functions, neighbourhoods, and penalty costs for finite set variables and constraints on these. We also introduced a number of set constraints and defined their penalty costs, and used these to model three combinatorial problems.

We believe that local search techniques are ready to be taken to a higher and more abstract level, and that one step in this direction is to introduce finite set variables. This will allow a user to utilise a modelling device that has been around for quite some time in the neighbouring constraint programming area. This will also mean that the designers and implementors of local search systems may reason about the components in their systems at a higher level. This should be beneficial for the necessary incremental algorithms and data structures, leading to more efficient systems.

\section{Acknowledgements}

I thank Pierre Flener and Justin Pearson for fruitful discussions and comments on earlier drafts of this paper.

\section{References}

1. Adam Ameur and Jakub Orzechowski Westholm. Local search methods in gene expression analysis. Technical Report T2002-12, Swedish Institute of Computer Science, 2002. Available from http://www.sics.se/libindex.html.

2. Markus Bohlin. Design and Implementation of a Graph-Based Constraint Model for Local Search, April 2004. PhL thesis, Department of Computer Science and Engineering, Mälardalen University, Västerås, Sweden. 
3. P. Codognet and D. Diaz. Yet another local search method for constraint solving. In K Steinhöfel, editor, Proceedings of SAGA 2001, First International Symposium on Stochastic Algorithms : Foundations and Applications, volume 2264 of LNCS, pages 73-90. Springer-Verlag, 2001.

4. Philippe Galinier and Jin-Kao Hao. Solving the progressive party problem by local search. In S. Voss, S. Martello, I.H. Osman, and C. Roucairol, editors, Metaheuristics: Advances and Trends in Local Search Paradigms for Optimization, chapter 29, pages 418-432. Kluwer Academic Publishers, 1998.

5. Philippe Galinier and Jin-Kao Hao. A general approach for constraint solving by local search. In Proceedings of CP-AI-OR'00, 2000.

6. Carmen Gervet. Set Intervals in Constraint Logic Programming: Definition and Implementation of a Language. PhD thesis, Université de Franche-Comté, France, September 1995. European thesis, in English.

7. Carmen Gervet. Interval propagation to reason about sets: Definition and implementation of a practical language. Constraints, 1(3):191-244, 1997.

8. Fred Glover and Manuel Laguna. Tabu search. In Modern Heuristic Techniques for Combinatorial Problems, pages 70-150. John Wiley \& Sons, 1993.

9. S. Kirkpatrick, C. D. Gelatt, Jr., and M. P. Vecchi. Optimization by simulated annealing. Science, 220(4598):671-680, may 1983.

10. Laurent Michel and Pascal Van Hentenryck. Localizer: A modeling language for local search. In Gert Smolka, editor, Proceedings of CP'97, volume 1330 of LNCS. Springer-Verlag, 1997.

11. Laurent Michel and Pascal Van Hentenryck. A constraint-based architecture for local search. ACM SIGPLAN Notices, 37(11):101-110, 2002. Proceedings of OOPSLA'02.

12. Laurent Michel and Pascal Van Hentenryck. Maintaining longest paths incrementally. In Francesca Rossi, editor, Proceedings of CP'03, volume 2833 of $L N C S$, pages 540-554. Springer-Verlag, 2003.

13. Tobias Müller and Martin Müller. Finite set constraints in Oz. In François Bry, Burkhard Freitag, and Dietmar Seipel, editors, 13. Workshop Logische Programmierung, pages 104-115, Technische Universität München, 17-19 September 1997.

14. Alexander Nareyek. Using global constraints for local search. In Proceedings of DIMACS Workshop on Constraint Programming and Large Scale Discrete Optimization, pages 1-18, 1998.

15. Bertrand Neveu and Gilles Trombettoni. When local search goes with the winners. In Michel Gendreau, Gilles Pesant, and Louis-Martin Rousseau, editors, Proceedings of CP-AI-OR'03, pages 180-194, 2003.

16. Jean-François Puget. Finite set intervals. In Proceedings of Workshop on Set Constraints, held at CP'96, 1996.

17. Pascal Van Hentenryck and Laurent Michel. Control abstractions for local search. In Francesca Rossi, editor, Proceedings of CP'03, volume 2833 of LNCS, pages 65-80. Springer-Verlag, 2003. 